Article

\title{
A Systematic Review of Studies Using the Brief COPE: Religious Coping in Factor Analyses
}

\author{
Christian U. Krägeloh
}

AUT University, Private Bag 92006, Auckland 1142, New Zealand;

E-Mail: chris.krageloh@aut.ac.nz; Tel.: +64-9-9219999 extension 7103; Fax: +64-9-9219780

Received: 23 May 2011; in revised form: 13 June 2011 / Accepted: 30 June 2011 /

Published: 4 July 2011

\begin{abstract}
Religion is generally recognized as a major resource for dealing with stressful events, but its relationship with secular coping strategies continues to be debated. The present article provides a systematic review of the way in which analyses of the sub-scale turning to religion of the widely used Brief COPE [1] instrument are presented in peer-reviewed research articles, in order to investigate how the wealth of data published using this instrument can inform how religious coping relates to other coping strategies. Of the 212 identified articles that included turning to religion in their analyses, 80 combined sub-scale scores to form higher-order coping factors, 38 of which based on exploratory factor analyses of their own datasets. When factor analyses had used individual items as indicators, religious coping was more likely to load together with maladaptive coping strategies, and more likely with adaptive coping strategies when analyses were conducted at sub-scale level. To a large extent, the variation in the results from exploratory factor analyses appears to be due to the diverse and often inappropriate factor analytic techniques used to determine the factor structure of the Brief COPE instrument. Reports from factor analyses of the Brief COPE therefore have very little value when trying to make general conclusions about the role of religious coping in relation to secular coping methods.
\end{abstract}

Keywords: coping; religion; religious coping; Brief COPE; systematic review

\section{Introduction}

By early adulthood, most people would have faced considerable stress at some point in their lives, and it is likely that they would have developed some behavior patterns that are intended to reduce the 
impact of stressors [2]. Such responses to environmental stressors are referred to as coping, which has been the subject of extensive investigations in psychological research during the past 40 years [3]. Although the specifics of the coping responses naturally vary across individuals, researchers have attempted to group similar types of responses into categories of coping strategies. An influential distinction was proposed by Lazarus and Folkman [4] and contrasts problem-focused with emotion-focused response strategies. While the former aim to modify the relationship between the environment and the person through dealing directly with the source of the stress, the latter attempt to regulate emotional distress by altering one's own response to the stressor.

What constitutes the most appropriate higher-order structure of coping is still being debated $[5,6]$, with researchers having put forward a number of alternative categorizations such as through the addition of avoidant coping [7,8] or dysfunctional coping [9]. At the lower-order level, in contrast, coping strategies are grouped into much more specific categories. Emotional support coping strategies, for example, could thus be defined as any set of responses emitted in the context of a stressful event that have the potential to mitigate the effects of stress by invoking the emotional support from other people, such as friends and family members. The variety of lower-order categories mentioned in the literature and used in empirical research is immense [6], and here the challenge is to determine which range of strategies and level of specificity allows for the most efficient assessment of people's use of coping responses. Coping is commonly assessed using self-report inventories, and questionnaire length is therefore a crucial practical consideration.

One frequently used coping questionnaire is the COPE [10], which, in its original format, assesses 13 lower-order strategies using four questions assigned to each of the following sub-scales: active coping, planning, suppression of competing activities, restraint coping, seeking social support for instrumental reasons, seeking social support for emotional reasons, positive reinterpretation and growth, acceptance, turning to religion, focus on and venting of emotions, denial, behavioral disengagement, and mental disengagement. The development of these sub-scales was conceptually driven, and the theoretical grouping of the items to their sub-scales was largely confirmed using principal-factors exploratory factor analysis. However, Carver et al. [10] did not provide detailed information about their factor analysis conducted in the development of the questionnaire, and the use of the Kaiser-Guttman criterion of selecting factors with eigenvalues above 1.0 may have overestimated the number of factors extracted [5,11]. When Carver et al. [10] conducted a secondorder factor analysis using the total sub-scale scores as variables, a four-factor structure emerged. Other researchers have since reported a three-factor structure $[9,12,13]$, or modified the questionnaire and proposed a five-factor structure [14]. It appears, therefore, that the structure of the COPE is considerably unstable, with results often highly dependent on the method of factor analysis employed [13].

Soon after the development of the COPE [10], Carver [1] published the Brief COPE, a shortened version of the COPE designed for use when participant response burden is a considering factor. This questionnaire asks 28 questions on a four-point Likert scale ("I haven't been doing this at all", "I've been doing this a little bit", "I've been doing this a medium amount", and "I've been doing this a lot"), where two items each form the following 14 sub-scales: active coping, planning, positive reframing, acceptance, humor, turning to religion, using emotional support, using instrumental support, selfdistraction, denial, venting, substance use, behavioral disengagement, and self-blame. Carver [1] 
developed this questionnaire based on a community sample of 168 participants who had been affected by a hurricane. Acknowledging the limitations from a factor analysis with a small sample size, Carver [1] nevertheless reported the factor structure obtained from item-level analyses. The only sub-scales that formed distinct factors were substance use, turning to religion, humor, and behavioral disengagement. The items from the remaining scales formed larger factors, but Carver [1] concluded that the structure approximated that of the full-length COPE questionnaire [10]. Rather than prescribing a rigid structure of the coping strategies assessed by the Brief COPE, Carver [1] recommended that researchers use the Brief COPE flexibly and creatively as suits, such as by suggesting the possibility of only selecting a sub-set of the sub-scales. Researchers using the Brief COPE therefore regularly refer to this recommendation to justify an exploratory analysis to determine empirically how the data from their sample is to be analyzed $[15,16]$.

The use of the variety of different Brief COPE [1] factor structures used in published research studies is part of the investigation of the present article. Focus will be given to the sub-scale turning to religion (the two items "I've been trying to find comfort in my religion or spiritual beliefs" and "I've been praying or meditating"), since this sub-scale is reported to behave in very diverse fashions in factor analyses. Schottenbauer et al. [17], for example, reported that the turning to religion sub-scale loaded onto a factor together with the sub-scales using emotional support and using instrumental support, and Farley et al. [18] found that turning to religion formed a factor together with the sub-sales positive reframing, acceptance, and humor. Religious coping forming its own distinct factor [19,20], or lack of sufficiently high loadings onto any other factor [21,22] is also commonly reported.

Religion and spirituality as a resource for coping has only recently received increasingly more attention [3]. While the benefits of religious involvement for both mental and physical health are well documented [23-25], its function as a resource for coping is still less well known. In a meta-analytic review of studies to investigate gender differences in coping, Tamres et al. [26] noted that religion could neither be clearly defined as problem- nor emotion-focused coping. Some researchers interpreted low factor loadings of religion with other coping strategies as indicating that it is a unique and independent strategy [27,28]. However, the concerns raised about commonly employed factor analytic methods with the COPE questionnaire [5,13] also apply to the Brief COPE and might help explain the highly variable reports of the position of religious coping in relation to other coping strategies.

Thus, the present study serves the following purposes: A systematic review of empirical studies will investigate how the religion sub-scale of the Brief COPE [1] is commonly analyzed. Studies will be identified that used factor structures that are based on previous research and those that conducted a factor analysis on their own dataset. Hereby, differences in methods of factor analyses used in the latter studies could reveal the reason for the variety of different reports of the behavior of the turning to religion sub-scale in relation to the factor structure of the remaining secular coping strategies. So far, no study has systematically explored the use of religious coping within the Brief COPE instrument, from which a pattern of the role of religious coping might emerge.

\section{Method}

In order to investigate how the turning to religion sub-scale of the Brief COPE [1] inventory is generally used in published research studies, a comprehensive literature search was conducted. Using 
the databases PsychInfo, Scopus, PubMed, Medline, and Google Scholar, peer-reviewed journal articles were identified that had referred to Carver [1] in their reference list and that were published until and including 2009. Access to the articles was obtained through the university library system of the author of the present article, through the inter-library loan service, or by requesting reprints directly from the authors of the concerning articles.

In total, 463 articles were identified and obtained in the manner described above. Only studies that had collected empirical data using the Brief COPE were considered for further analyses, of which there were 399. Review articles and commentaries were thus excluded. Given that the focus of the present study was on religious coping, studies that did not collect data of the turning to religion sub-scale or used substantially modified items were discarded, reducing the number of articles to 290. Further 78 studies were excluded as they that did not provide enough information about which items in the Brief COPE were used or in which manner the scores were calculated. The information extracted from the remaining 212 articles was the manner in which the religion sub-scales was used, such as whether scores from the religion items were presented individually or as a sub-scale total, or whether the items contributed to a score that was calculated by summation of item scores from a number of different coping strategies. In the latter case, it was determined whether the calculation of scores was based on the findings from previous research or whether it was based on the relevant study's own exploratory factor analysis. If a factor analysis was conducted, the main factor analytic method used was identified, as well as how turning to religion loaded to the proposed factor structure. Although principal components analysis (PCA) is technically not considered as a type of factor analysis, for ease of presentation of the present results, references to the term factor analysis also include PCA.

\section{Results}

Of the 212 empirical studies that had used the Brief COPE [1] and fit the criteria mentioned above, more than half $(N=125)$ had used scores from the individual sub-scales. Four studies presented analyses using scores from individual items [29-32], and three studies used total scores [33-35]. The number of studies that had used scores calculated based on a factor structure was 80, of which 38 studies had conducted an exploratory factor analysis on their own dataset.

\subsection{Studies that conducted their own exploratory factor analysis}

Tables 1 and 2 show a summary of the studies that conducted their own exploratory factor analysis at item and sub-scale level, respectively. The majority of studies explicitly stated whether they had conducted their analyses at item level or by treating sub-scale totals as indicators. No study mentioned having conducted a higher-order factor analysis to test for a lower-order factor structure and how these lower-order factors are correlated with higher-order factors [36]. With 14 theoretical sub-scales, such a model would have been very complex, requiring such a large sample size that it is unlikely that researchers would have attempted this type of analysis. Instead, many authors referred to the term high-order factor analysis and explained it as conducting a factor analysis at sub-scale level, as used by Carver et al. [10] in the development of the COPE questionnaire. Thus, when authors simply stated that they had conducted a higher-order factor analysis without providing more detail, it was assumed that this referred to using sub-scales as indicators, although the more appropriate technical term for this 
analysis would have been individual extension analysis [37]. It is highly likely that this assumption was warranted, since the reported factor loadings in those cases were always reported for sub-scales only, and never for individual items.

The summary of results from item-level factor analyses are grouped separately in Table 1, depending on whether studies reported that the two religious coping items loaded together with items from other sub-scales, formed an independent factor, or whether they failed to load. In cases where two separate papers reported on different aspects of the same dataset, the study was included in the table that provided the most amount of detail about its methods. This concerned the studies by Brain $e t$ al. [19] and Henderson et al. [38], as well as Radat et al. [15] and Lucas et al. [39], where the results from Henderson et al. and Lucas et al. are not shown.

As shown in Table 1, only one study reported that the turning to religion items did not fit onto the proposed factor structure [22]. Five studies [16,40-43] reported that items from the religious coping sub-scale loaded together with items from other sub-scales. For four of these studies, both turning to religion items loaded onto the same factor, whereas in Zelikovsky et al. [16] only one item loaded with another factor, while the other item was excluded due to a factor loading below 0.40. All five studies conducted a PCA. Three of these [16,40,41] used an orthogonal (varimax) rotation, one [42] an oblique (direct oblimin) rotation, and one study [43] did not specify the rotation method. Apart from the study by Lee and Liu [41], who had a sample size of 406 using university undergraduate students, the sample sizes of the other four studies were very similar, ranging from 104 to 154 . According to the commonly stated rule of thumb of five participants per indicator [44], this could be considered insufficient to marginal for a 28 -item questionnaire.

Ten of the studies $[15,19,20,45-51]$ that conducted item-level analyses reported that the two items from the turning to religion sub-scale formed their own separate factor (Table 1). Three of these studies [20,49,50] used a sample of university students, but overall, the range was very broad, and included diverse participants groups, such as emergency workers [45], edentulous patients with complete dentures [48], or genetic counselors [51]. PCA was the stated method of choice for four of the ten studies $[15,19,46,50]$. Three studies $[20,45,48]$ only stated that they had conducted a factor analysis without further specification, two used a principal axis factoring technique [47,51], and one stated that they had used a robust weighted least squares technique with a polychoric correlation matrix [49]. Compared to the five studies that had reported that religion loaded onto a factor with other coping strategies, the ten studies that found religion to form a separate factor used considerably larger sample sizes, ranging from 132 [46] to 1534 [15], with a mean of 587.80.

Table 2 shows the summaries of the studies that had conducted exploratory factor analyses using the sub-scale total scores as indicators. As with the item-level analyses, the samples were very diverse, and the sample sizes varied from 71 [52] to 1289 [17], with a mean of 288.70. Twelve studies [17,18,52-61] reported that the turning to religion sub-scale loaded to a factor with at least one other sub-scale, three studies [62-64] reported that the turning to religion sub-scale formed its own separate factor, and five studies [21,65-68] reported that the religion sub-scale did not have a sufficiently high loading with any other factor. As with the item-level analyses (Table 1), PCA was the most frequently used technique, with 9 of the 20 studies [18,52-54,56,57,62,64,66] explicitly stating this method. Of the ten studies that mentioned the rotation method used, nine [18,21,52-55,61,62,67] stated varimax, and one stated that they had used an oblique rotation [68]. Nine studies $[17,21,58-60,63,65,67,68]$ only 
stated that they had used a factor analysis or exploratory factor analysis, and two studies had used a principal axis factor analysis [55,61]. Compared to item-level analyses, where the median number of extracted factors was six, the median number of factors extracted in studies using sub-scale level exploratory factor analyses was three. In the calculation of the median, the two-factor solution by Kershaw et al. [58] was not included, as they reported that the religion sub-scale loaded differently, depending on whether data from caregivers or care recipients were considered.

\subsection{Studies that did not conduct their own exploratory factor analysis}

The studies that used factor structures based on theoretical considerations or factor structures based on previous research are listed in Table 3. As with Tables 1 and 2, when at least two studies reported results based on the same dataset, only summaries are shown for the study that provided the most detail. Summaries for the following eight studies are therefore not listed in Table 3: Cooper et al. [69-71], Gillen [72], Gore-Felton et al. [73], Gow et al. [74], Pence et al. [75], and Piazza-Waggoner et al. [76]. Compared to the studies that had conducted their own exploratory factor analyses, those that based their factor structure on previous research tended to have smaller samples sizes. Of the 34 studies listed in Table 3, 14 studies had a sample size of less than 100 [77-90]. Some of the studies had more than 300 participants [91-93], where a factor analysis would have been supported by a sufficiently large sample size.

With a median number of two factors, studies that used a pre-existing factor structure tended to use a factor structure at the lower end of the numbers reported from exploratory factor analyses (Tables 1 and 2). Seven of the 34 studies listed in Table 3 [79,80,85,92,94-96] referred to Carver et al. [10] to justify the use of their factor structure, and two [89,92] to Carver [1]. Four studies [88,91,97,98] provided some general theoretical or practical considerations, and eight studies [81,93,99-104] did not provide any justification or reference. The remaining studies referred to a variety of studies using either the COPE or Brief COPE questionnaire. Atkinson et al. [77] referred to Lunsford et al. [105], although Lunsford et al. themselves used the separate Brief COPE sub-scale scores. Some studies referred to the theoretical considerations from other researchers, such as Cartwright et al. [106] referring to Schnider et al. [98].

\subsection{Overview of findings}

Table 4 provides a summary of the factor loading pattern of the Brief COPE turning to religion items, presented separately by results from exploratory factor analyses at item level (Table 1), sub-scale level (Table 2), and, for comparative purposes, of the studies that used factor structures based on previous research (Table 3). Artinian et al. [94], for example, reported that the turning to religion sub-scale loaded together with the sub-scales active coping, planning, positive reframing, acceptance, humor, emotional support, instrumental support, self-distraction, and venting. The entry "1" was therefore made under the respective columns in Table 4. Entries were made in this fashion for all studies shown in Tables 1 to 3. For item-level analyses, split-loading was possible, such as reported by Lee and Liu [41] and Zelikovsky et al. [16]. In the case of Lee and Liu [41], the two items from the turning to religion sub-scale loaded together with only one item each from humor and venting, and therefore "0.5" was entered for humor and venting. Zelikovsky et al. [16] reported that only one of the 
items from the turning to religion sub-scale loaded onto a factor, and therefore the entry was " 0.5 " when it loaded with two items from another sub-scale (behavioral disengagement, denial, substance use, and self-blame) or " 0.25 " when it loaded with only one item from another sub-scale (humor and venting). Data for Kershaw et al. [58] are not shown in Table 4, since turning to religion loaded onto a different factor depending on whether the exploratory factor analysis was conducted for caregivers or care recipients.

The percentage of cases that turning to religion loaded with another coping strategy was then calculated. For sub-scale-level analyses, for example, turning to religion loaded together with active coping in seven of eleven studies (64\%). Comparing the results between item and sub-scale levels, some interesting differences emerge. For item-level analyses, turning to religion appeared to load more often with the strategies venting, behavioral disengagement, denial, substance use, and self-blame, whereas for sub-scale level analyses, turning to religion tended to load together with active coping, planning, positive reframing, acceptance, humor, emotional support, instrumental support, and self-distraction. The loading pattern for studies using factor structures based on previous studies was similar to those from the sub-scale factor analyses. The most obvious exception was humor, which was used in a factor together with turning to religion in $71 \%$ of the studies that based their factor structure on previous research, when only $15 \%$ of item-level analyses and 18\% of sub-scale-level analyses reported that religious coping loaded together with humor.

\section{Discussion}

\subsection{General discussion}

The present study provided a systematic review of the way in which religious coping is assessed in peer-reviewed research studies using the Brief COPE [1] inventory. Encouraged by the recommendations of the inventory's author, researchers often conduct a factor analysis on their dataset to determine the way in which the scores from the 14 coping sub-scales are to be calculated for their sample. The present review analyzed 16 studies that had conducted an exploratory factor analysis at item level and 20 studies that reported results from analyses conducted at sub-scale level. The most commonly used method in both cases was PCA, and the diversity of the sample population of the individual studies was comparable. The most striking difference in the results between the two types of approaches to exploratory factor analysis was that, when the analyses were conducted at sub-scale level, turning to religion more commonly loaded onto a factor with strategies such as active coping and positive reframing, or strategies sometimes referred to as adaptive [83], as opposed to item-level analyses, where religious coping was more likely to load with maladaptive strategies, such as behavioral disengagement or denial (Table 4). In 10 of 16 cases, religion formed its own factor for item-level analyses (Table 1), compared to only 3 of 20 cases for sub-scale-level analyses (Table 2). Factor analyses at sub-scale level were more likely to report a lack of factor loading for religious coping, with 5 out of 20 cases, compared to 1 out of 16 factor analyses at item level, although Zelikovsky et al. [16] reported that one of the religious coping items did not load, either. 
Table 1. Summary of studies identified in the systematic review that had conducted an item-level factor analysis on their own dataset. Listed are the type of sample, the sample size, the method of factor analysis used, and whether items had been excluded from the factor analysis. The number of factors extracted is also listed for each study. Unless otherwise stated in the notes, an English language version of the Brief COPE [1] was used.

\begin{tabular}{|c|c|c|c|c|c|}
\hline Author & Sample & $\begin{array}{l}\text { Sample } \\
\text { Size }\end{array}$ & $\begin{array}{l}\text { Method of exploratory } \\
\text { factor analysis }\end{array}$ & $\begin{array}{l}\text { Excluded items prior to } \\
\text { factor analysis }\end{array}$ & $\begin{array}{l}\text { Number of Notes } \\
\text { factors } \\
\text { extracted }\end{array}$ \\
\hline \multicolumn{6}{|c|}{$\begin{array}{l}\text { Religious coping loading together with } \\
\text { items from other sub-scales: }\end{array}$} \\
\hline Hastings et al. [40] & $\begin{array}{l}\text { Parents of children } \\
\text { with autism }\end{array}$ & 135 & $\begin{array}{l}\text { Principal components } \\
\text { analysis with varimax } \\
\text { rotation }\end{array}$ & None & 4 \\
\hline Lee and Liu [41] & $\begin{array}{l}\text { University } \\
\text { undergraduate } \\
\text { students }\end{array}$ & 406 & $\begin{array}{l}\text { Principal components } \\
\text { analysis with varimax } \\
\text { rotation }\end{array}$ & $\begin{array}{l}\text { Two items each from the } \\
\text { sub-scales instrumental } \\
\text { support and self-blame }\end{array}$ & 2 \\
\hline $\begin{array}{l}\text { Liu and Iwamoto } \\
\text { [42] }\end{array}$ & University students & 154 & $\begin{array}{l}\text { Principal components } \\
\text { analysis with direct oblimin } \\
\text { rotation }\end{array}$ & $\begin{array}{l}\text { Two items from the sub-scale } \\
\text { substance use }\end{array}$ & 2 \\
\hline Paukert et al. [43] & $\begin{array}{l}\text { Heart failure } \\
\text { patients }\end{array}$ & 104 & $\begin{array}{l}\text { Principal components } \\
\text { analysis }\end{array}$ & None & 3 \\
\hline $\begin{array}{l}\text { Zelikovsky et al. } \\
{[16]}\end{array}$ & $\begin{array}{l}\text { Parents of children } \\
\text { with end-stage } \\
\text { renal disease }\end{array}$ & 144 & $\begin{array}{l}\text { Principal components } \\
\text { analysis with varimax } \\
\text { rotation }\end{array}$ & None & 2 \\
\hline \multicolumn{6}{|c|}{$\begin{array}{l}\text { Religious coping items forming separate } \\
\text { factor: }\end{array}$} \\
\hline Brain et al. [19] & $\begin{array}{l}\text { Young women with } \\
\text { breast cancer }\end{array}$ & 1286 & $\begin{array}{l}\text { Principal components } \\
\text { analysis }\end{array}$ & $\begin{array}{l}\text { Two items, not mentioned } \\
\text { which ones }\end{array}$ & 7 \\
\hline
\end{tabular}


Table 1. Cont.

Cicognani et al. [45] Emergency workers

764

Women undergoing

Fillion et al. [46]

radiation therapy

132

for breast cancer

Relatives of newly

Fletcher et al. [47] diagnosed breast cancer patients

Edentulous patients

Heydecke et al. [48] with complete dentures

Miyazaki et al. [49]

International university students

University

Perczek et al. [20]

undergraduate

students

Adult migraine

Radat et al. [15] sufferers from the

general population
Two items each from the

sub-scales behavioral

disengagement, denial, and

substance use

Principal components analysis with oblique rotation

None

Added items and modified items slightly

Two items each from the

sub-scales planning, humor, behavioral disengagement,

substance use and self-blame

Factor analysis with oblique rotation

None

Robust weighted least squares with polychoric correlation

None

matrix with promax rotation

None

rotation

Principal components

analysis with varimax

rotation of the correlation

None

matrix
Conducted factor analysis with items from both versions together

French language version Spanish and English 
Table 1. Cont.

\begin{tabular}{|c|c|c|c|c|c|c|}
\hline $\begin{array}{l}\text { Ribeiro and } \\
\text { Rodrigues [50] }\end{array}$ & $\begin{array}{l}\text { University } \\
\text { undergraduate } \\
\text { students }\end{array}$ & 364 & $\begin{array}{l}\text { Principal components } \\
\text { analysis with oblique rotation }\end{array}$ & None & 8 & $\begin{array}{l}\text { Portuguese language } \\
\text { version }\end{array}$ \\
\hline Udipi et al. [51] & Genetic counselors & 222 & $\begin{array}{l}\text { Principal axis factor analysis } \\
\text { with varimax rotation }\end{array}$ & None & 8 & $\begin{array}{l}\text { Some minor modifications } \\
\text { to items }\end{array}$ \\
\hline \multicolumn{7}{|c|}{$\begin{array}{l}\text { Religious coping items not loading onto a } \\
\text { factor: }\end{array}$} \\
\hline $\begin{array}{l}\text { Welbourne et al. } \\
\text { [22] }\end{array}$ & Nurses & 190 & $\begin{array}{l}\text { Principal component factor } \\
\text { analysis with varimax } \\
\text { rotation }\end{array}$ & None & 3 & $\begin{array}{l}\text { Religion items not loading } \\
\text { onto a factor, with loading } \\
<0.40\end{array}$ \\
\hline
\end{tabular}

Table 2. Summary of studies identified in the systematic review that had conducted a sub-scale-level factor analysis on their own dataset. Listed are the type of sample, the sample size, the method of factor analysis used, and whether items had been excluded from the factor analysis. The number of factors extracted is also listed for each study. Unless otherwise stated in the notes, an English language version of the Brief COPE [1] was used.

\begin{tabular}{|c|c|c|c|c|c|c|}
\hline Author & Sample & $\begin{array}{l}\text { Sample } \\
\text { Size }\end{array}$ & $\begin{array}{l}\text { Method of exploratory factor } \\
\text { analysis }\end{array}$ & $\begin{array}{l}\text { Excluded sub-scales } \\
\text { prior to factor } \\
\text { analysis }\end{array}$ & $\begin{array}{l}\text { Number } \\
\text { of factors } \\
\text { extracted }\end{array}$ & Notes \\
\hline \multicolumn{7}{|c|}{$\begin{array}{l}\text { Religious coping loading together with } \\
\text { items from other sub-scales: }\end{array}$} \\
\hline $\begin{array}{l}\text { Aitken and } \\
\text { Crawford [52] }\end{array}$ & Project managers & 71 & $\begin{array}{l}\text { Principal components analysis } \\
\text { with varimax rotation }\end{array}$ & None & 2 & $\begin{array}{l}\text { The sub-scales instrumental } \\
\text { support and self-blame loaded } \\
\text { onto both factors }\end{array}$ \\
\hline Bean et al. [53] & $\begin{array}{l}\text { Patients with heart } \\
\text { failure }\end{array}$ & 100 & $\begin{array}{l}\text { Principal components analysis } \\
\text { with varimax rotation }\end{array}$ & None & 2 & \\
\hline $\begin{array}{l}\text { Bellizzi and Blank } \\
\text { [54] }\end{array}$ & $\begin{array}{l}\text { Breast cancer } \\
\text { survivors }\end{array}$ & 224 & $\begin{array}{l}\text { Principal components analysis } \\
\text { with varimax rotation }\end{array}$ & None & 2 & \\
\hline
\end{tabular}


Table 2. Cont.

\begin{tabular}{|c|c|c|c|c|c|c|}
\hline Ebert et al. [55] & $\begin{array}{l}\text { University } \\
\text { undergraduate } \\
\text { students }\end{array}$ & 202 & $\begin{array}{l}\text { Principal factor analysis with } \\
\text { varimax rotation }\end{array}$ & None & 2 & \\
\hline Farley et al. [18] & $\begin{array}{l}\text { Rural population in } \\
\text { USA }\end{array}$ & 288 & $\begin{array}{l}\text { Principal components analysis } \\
\text { with varimax rotation }\end{array}$ & None & 4 & \\
\hline Jacobson [56] & $\begin{array}{l}\text { Employee assistance } \\
\text { professionals }\end{array}$ & 325 & Principal components analysis & None & 3 & \\
\hline Kellezi et al. [57] & $\begin{array}{l}\text { Kosovo Albanians } \\
\text { who experienced } \\
\text { extreme events in } \\
\text { war }\end{array}$ & 127 & Principal components analysis & None & 1 & $\begin{array}{l}\text { Albanian language version; } \\
\text { forced a one-factor solution } \\
\text { and excluded items that did } \\
\text { not load }\end{array}$ \\
\hline Kershaw et al. [58] & $\begin{array}{l}\text { Patients with breast } \\
\text { cancer and their } \\
\text { caregivers }\end{array}$ & 189 & Exploratory factor analysis & $\begin{array}{l}\text { Instrumental support } \\
\text { and self-blame }\end{array}$ & 2 & $\begin{array}{l}\text { For family caregivers, loaded } \\
\text { with humor, self-distraction, } \\
\text { venting, behavioral } \\
\text { disengagement, denial, and } \\
\text { substance use. For patients, } \\
\text { with active coping, planning, } \\
\text { positive reframing, } \\
\text { acceptance, and emotional } \\
\text { support }\end{array}$ \\
\hline Liu et al. [59] & $\begin{array}{l}\text { Renal transplant } \\
\text { recipients }\end{array}$ & 160 & Factor analysis & Substance use & 2 & \\
\hline $\begin{array}{l}\text { Mugavero et al. } \\
{[60]}\end{array}$ & $\begin{array}{l}\text { Patients infected with } \\
\text { HIV }\end{array}$ & 611 & Exploratory factor analysis & $\begin{array}{l}\text { Planning, humor, } \\
\text { instrumental support, } \\
\text { self-distraction, } \\
\text { venting, and substance } \\
\text { use }\end{array}$ & 2 & $\begin{array}{l}\text { Not mentioned whether the } \\
\text { sub-scales were excluded } \\
\text { before the exploratory factor } \\
\text { analysis or as a result of it }\end{array}$ \\
\hline
\end{tabular}


Table 2. Cont

\begin{tabular}{|c|c|c|c|c|c|c|}
\hline $\begin{array}{l}\text { Schottenbauer } \\
\text { et al. [17] }\end{array}$ & $\begin{array}{l}\text { Participants } \\
\text { responding to an } \\
\text { internet survey }\end{array}$ & 1289 & Exploratory factor analysis & None & 3 & $\begin{array}{l}\text { Religion was later dropped } \\
\text { due to overlap with other } \\
\text { measures }\end{array}$ \\
\hline $\begin{array}{l}\text { Steinhardt and } \\
\text { Dolbier [61] }\end{array}$ & $\begin{array}{l}\text { University } \\
\text { undergraduate } \\
\text { students }\end{array}$ & 114 & $\begin{array}{l}\text { Principal axis factor analysis } \\
\text { with varimax rotation }\end{array}$ & None & 4 & $\begin{array}{l}\text { Loaded with positive } \\
\text { reframing and substance use } \\
\text { (negatively scored) }\end{array}$ \\
\hline \multicolumn{7}{|c|}{$\begin{array}{l}\text { Religious coping sub-scale forming } \\
\text { separate factor: }\end{array}$} \\
\hline $\begin{array}{l}\text { Saroglou and } \\
\text { Anciaux [62] }\end{array}$ & $\begin{array}{l}\text { Convenience sample } \\
\text { of adults }\end{array}$ & 256 & $\begin{array}{l}\text { Principal components analysis } \\
\text { with varimax rotation }\end{array}$ & None & 5 & $\begin{array}{l}\text { French language version; with } \\
\text { high cross-loading of } \\
\text { behavioral disengagement } \\
\text { and negatively scored humor }\end{array}$ \\
\hline $\begin{array}{l}\text { Weininger et al. } \\
\text { [63] }\end{array}$ & Physicians & 212 & Factor analysis & $\begin{array}{l}\text { Added two items about } \\
\text { political activity }\end{array}$ & 4 & $\begin{array}{l}\text { Not entirely clear whether } \\
\text { conducted at item or sub-scale } \\
\text { level }\end{array}$ \\
\hline $\begin{array}{l}\text { Wood and } \\
\text { Rutterford [64] }\end{array}$ & $\begin{array}{l}\text { People recovering } \\
\text { from brain injury }\end{array}$ & 131 & $\begin{array}{l}\text { Principal components analysis } \\
\text { using the correlation matrix }\end{array}$ & None & 5 & \\
\hline \multicolumn{7}{|c|}{$\begin{array}{l}\text { Religious coping sub-scale not loading } \\
\text { onto a factor: }\end{array}$} \\
\hline Kershaw et al. [65] & $\begin{array}{l}\text { Prostate cancer } \\
\text { patients and spouses }\end{array}$ & 121 & Exploratory factor analysis & None & 2 & $\begin{array}{l}\text { Religion sub-scale not loading } \\
\text { onto a factor, with loading } \\
<0.40 \text {, or having factor } \\
\text { loading of }>0.40 \text { on two } \\
\text { factors (not specified) }\end{array}$ \\
\hline $\begin{array}{l}\text { Lawrence and } \\
\text { Fauerbach [21] }\end{array}$ & $\begin{array}{l}\text { Adult burn survivors } \\
\text { requiring } \\
\text { hospitalization }\end{array}$ & 158 & $\begin{array}{l}\text { Exploratory factor analysis with } \\
\text { least square extraction method } \\
\text { and varimax rotation }\end{array}$ & $\begin{array}{l}\text { Instrumental support, } \\
\text { substance use, and } \\
\text { self-blame }\end{array}$ & 2 & $\begin{array}{l}\text { Religion sub-scale not loading } \\
\text { onto a factor, with loading } \\
<0.30\end{array}$ \\
\hline
\end{tabular}


Table 2. Cont.

\begin{tabular}{|c|c|c|c|c|c|c|}
\hline $\begin{array}{l}\text { Myaskovsky et al. } \\
\text { [66] }\end{array}$ & $\begin{array}{l}\text { Adult lung transplant } \\
\text { candidates }\end{array}$ & 114 & Principal components analysis & None & 5 & $\begin{array}{l}\text { Although not explicitly stated, } \\
\text { appears that religion did not } \\
\text { load }\end{array}$ \\
\hline Ng and Leung [67] & $\begin{array}{l}\text { Dental practice } \\
\text { patients }\end{array}$ & 1000 & $\begin{array}{l}\text { Factor analysis with varimax } \\
\text { rotation }\end{array}$ & None & 3 & $\begin{array}{l}\text { Although not explicitly stated, } \\
\text { appears that religion did not } \\
\text { load }\end{array}$ \\
\hline Yang et al. [68] & $\begin{array}{l}\text { Women diagnosed } \\
\text { with breast cancer }\end{array}$ & 82 & $\begin{array}{l}\text { Exploratory factor analysis with } \\
\text { oblique rotation }\end{array}$ & Self-blame & 2 & $\begin{array}{l}\text { Religion sub-scale not loading } \\
\text { onto a factor, with loading } \\
<0.30\end{array}$ \\
\hline
\end{tabular}

Table 3. Summary of studies identified in the systematic review that had used a factor structure of the Brief COPE [1] that was based on previous research. Listed are the type of sample, the sample size, the justification provided for using the factor structure, and whether subscales had been excluded from the study. The number of factors extracted is also listed for each study. Unless otherwise stated in the notes, an English language version of the Brief COPE was used.

\begin{tabular}{|c|c|c|c|c|c|c|}
\hline Author & Sample & $\begin{array}{l}\text { Sample } \\
\text { Size }\end{array}$ & $\begin{array}{l}\text { Justification of factor } \\
\text { structure }\end{array}$ & Excluded sub-scales & $\begin{array}{l}\text { Number } \\
\text { of factors }\end{array}$ & Notes \\
\hline Artinian et al. [94] & $\begin{array}{l}\text { Patients in cardiac } \\
\text { rehabilitation program }\end{array}$ & 112 & Carver et al. [10] & None & 2 & \\
\hline Atkinson et al. [77] & $\begin{array}{l}\text { Adults with HIV } \\
\text { infection }\end{array}$ & 34 & Lunsford et al. [105] & None & 2 & $\begin{array}{l}\text { Lunsford et al. [63] used } \\
\text { scores from individual } \\
\text { sub-scales }\end{array}$ \\
\hline Cartwright et al. [106] & $\begin{array}{l}\text { Individuals with } \\
\text { alopecia recruited from } \\
\text { internet support groups }\end{array}$ & 214 & Schnider et al. [98] & None & 3 & \\
\hline Cooper et al. [107] & $\begin{array}{l}\text { Family caregivers of } \\
\text { patients with } \\
\text { Alzheimer's disease }\end{array}$ & 126 & Coolidge et al. [9] & None & 3 & \\
\hline
\end{tabular}


Table 3. Cont.

\begin{tabular}{|c|c|c|c|c|c|c|}
\hline David and Knight [91] & $\begin{array}{l}\text { Adults self-identified as } \\
\text { gay }\end{array}$ & 383 & $\begin{array}{l}\text { Consistent with previous use, } \\
\text { but no reference provided }\end{array}$ & None & 2 & $\begin{array}{l}\text { Conducted confirmatory } \\
\text { factor analysis }\end{array}$ \\
\hline Gillen [78] & Stroke survivors & 16 & Meyer [83] & None & 2 & \\
\hline Glass et al. [108] & Hurricane survivors & 228 & Schnider et al. [98] & None & 2 & \\
\hline Gore-Felton et al. [97] & $\begin{array}{l}\text { HIV positive and AIDS } \\
\text { patients }\end{array}$ & 122 & $\begin{array}{l}\text { Theoretical considerations, } \\
\text { although not much } \\
\text { information provided }\end{array}$ & $\begin{array}{l}\text { Humor, instrumental } \\
\text { support, self-distraction, } \\
\text { venting, behavioral } \\
\text { disengagement, substance } \\
\text { use, self-blame }\end{array}$ & 2 & \\
\hline Gow et al. [92] & Male apprentices & 326 & Carver et al. [10]; Carver [1] & None & 3 & \\
\hline Hart et al. [99] & $\begin{array}{l}\text { HIV positive and AIDS } \\
\text { patients }\end{array}$ & 105 & $\begin{array}{l}\text { No justification or reference } \\
\text { provided }\end{array}$ & $\begin{array}{l}\text { Instrumental support and } \\
\text { self-blame }\end{array}$ & 2 & $\begin{array}{l}\text { Mentioned that the other } \\
\text { factor consisted of active } \\
\text { coping and planning, but } \\
\text { did not mention about the } \\
\text { remaining sub-scales }\end{array}$ \\
\hline $\begin{array}{l}\text { Hastings and Brown } \\
\text { [79] }\end{array}$ & $\begin{array}{l}\text { Staff members working } \\
\text { at a school for children } \\
\text { with developmental } \\
\text { disabilities }\end{array}$ & 55 & Carver et al. [10] & & 2 & \\
\hline Hirsch et al. [109] & Pharmacy students & 213 & Meyer [83] & No information & 2 & \\
\hline $\begin{array}{l}\text { Ironson and Kremer } \\
{[100]}\end{array}$ & Individuals with HIV & 147 & $\begin{array}{l}\text { No justification or reference } \\
\text { provided }\end{array}$ & $\begin{array}{l}\text { Humor, instrumental } \\
\text { support, venting, and self- } \\
\text { blame }\end{array}$ & 2 & \\
\hline Kinsinger et al. [101] & $\begin{array}{l}\text { Men receiving } \\
\text { treatment for prostate } \\
\text { cancer }\end{array}$ & 250 & $\begin{array}{l}\text { No justification or reference } \\
\text { provided }\end{array}$ & $\begin{array}{l}\text { Some sub-scales } \\
\text { excluded, but not } \\
\text { mentioned which ones }\end{array}$ & 2 & \\
\hline
\end{tabular}


Table 3. Cont

\begin{tabular}{|c|c|c|c|c|c|c|}
\hline Knoll et al. [95] & Cataract patients & 110 & Carver et al. [10] & $\begin{array}{l}\text { Self-distraction, } \\
\text { behavioral disengagement, } \\
\text { and substance use }\end{array}$ & 4 & $\begin{array}{l}\text { German language version; } \\
\text { conducted a confirmatory } \\
\text { factor analysis }\end{array}$ \\
\hline Kristiansen et al. [80] & Elite wrestlers & 82 & $\begin{array}{l}\text { Carver et al. [10]; Hastings } \\
\text { and Brown [79] }\end{array}$ & Positive reframing & 2 & $\begin{array}{l}\text { Norwegian language } \\
\text { version }\end{array}$ \\
\hline Krzemien [96] & $\begin{array}{l}\text { Women between } 60 \text { to } \\
95 \text { years of age }\end{array}$ & 212 & Carver et al. [10] & None & 3 & Spanish language version \\
\hline Lethborg et al. [102] & $\begin{array}{l}\text { Oncology clinic } \\
\text { patients }\end{array}$ & 100 & $\begin{array}{l}\text { No justification or reference } \\
\text { provided }\end{array}$ & None & 2 & \\
\hline $\begin{array}{l}\text { Lord and Robertson } \\
\text { [81] }\end{array}$ & $\begin{array}{l}\text { Patients at assisted } \\
\text { conception units }\end{array}$ & 50 & $\begin{array}{l}\text { No justification reference } \\
\text { provided }\end{array}$ & None & 2 & \\
\hline McIlvane et al. [82] & $\begin{array}{l}\text { Individuals recently } \\
\text { diagnosed with mild } \\
\text { cognitive impairment } \\
\text { and their care partners }\end{array}$ & 75 & $\begin{array}{l}\text { Cooper et al. [69], Coolidge } \\
\text { et al. [9] }\end{array}$ & None & 3 & \\
\hline Meyer [83] & $\begin{array}{l}\text { Psychiatric ward } \\
\text { inpatients }\end{array}$ & 70 & Carver et al. [112] & None & 2 & \\
\hline $\begin{array}{l}\text { Moscardino et al. } \\
{[110]}\end{array}$ & $\begin{array}{l}\text { Adolescent school } \\
\text { children who } \\
\text { experiences the Beslan } \\
\text { terrorist attacks }\end{array}$ & 171 & $\begin{array}{l}\text { Feaster and Szapocznik } \\
{[113]}\end{array}$ & None & 3 & $\begin{array}{l}\text { Conducted confirmatory } \\
\text { factor analysis }\end{array}$ \\
\hline O'Brien et al. [84] & $\begin{array}{l}\text { Adolescents considered } \\
\text { high risk of developing } \\
\text { psychosis }\end{array}$ & 16 & $\begin{array}{l}\text { The selected strategies were } \\
\text { encouraged during the } \\
\text { treatment group }\end{array}$ & $\begin{array}{l}\text { Venting, denial, } \\
\text { behavioral disengagement, } \\
\text { substance use, and self- } \\
\text { blame }\end{array}$ & 1 & \\
\hline O’Connor et al. [85] & $\begin{array}{l}\text { End-stage renal disease } \\
\text { patient }\end{array}$ & 73 & Carver et al. [10] & $\begin{array}{l}\text { Denial, venting, substance } \\
\text { use, behavioral } \\
\text { disengagement, and self- } \\
\text { blame }\end{array}$ & 3 & \\
\hline
\end{tabular}


Table 3. Cont.

\begin{tabular}{|c|c|c|c|c|c|c|}
\hline $\begin{array}{l}\text { Piazza-Waggoner } \\
\text { et al. }[86]\end{array}$ & $\begin{array}{l}\text { Patients at a university- } \\
\text { based pediatric clinic }\end{array}$ & 64 & Spirito [114] & None & 2 & \\
\hline Richards et al. [103] & $\begin{array}{l}\text { Polymorphic light } \\
\text { eruption patients }\end{array}$ & 145 & $\begin{array}{l}\text { No justification or reference } \\
\text { provided }\end{array}$ & None & 2 & \\
\hline Rosenberger et al. [87] & $\begin{array}{l}\text { Patients undergoing } \\
\text { knee arthroscopic } \\
\text { surgery }\end{array}$ & 81 & Stephens et al. [115] & $\begin{array}{l}\text { Instrumental support and } \\
\text { self-blame }\end{array}$ & 2 & $\begin{array}{l}\text { Also used individual sub- } \\
\text { scale data }\end{array}$ \\
\hline Schnider et al. [98] & University students & 123 & Theoretical considerations & None & 3 & \\
\hline Schwartz et al. [104] & $\begin{array}{l}\text { Urban minority } \\
\text { participants diagnosed } \\
\text { with chlamydia or } \\
\text { gonorrhoea }\end{array}$ & 259 & $\begin{array}{l}\text { No justification or reference } \\
\text { provided }\end{array}$ & $\begin{array}{l}\text { Positive reframing, } \\
\text { acceptance, humor, self- } \\
\text { distraction, venting, and } \\
\text { substance use }\end{array}$ & 2 & \\
\hline Steinhardt et al. [88] & $\begin{array}{l}\text { African Americans with } \\
\text { Type } 2 \text { diabestes }\end{array}$ & 16 & Theoretical considerations & None & 2 & \\
\hline $\begin{array}{l}\text { Straughan and } \\
\text { Buckenham [89] }\end{array}$ & $\begin{array}{l}\text { Outpatients diagnosed } \\
\text { with bipolar disorder }\end{array}$ & 48 & Carver [1] & None & 2 & \\
\hline Tuncay et al. [111] & $\begin{array}{l}\text { Patients with Type } 1 \\
\text { and } 2 \text { diabetes }\end{array}$ & 161 & Tuna [116] & None & 2 & Turkish language version \\
\hline Wichianson et al. [90] & College students & 95 & Meyer [83] & None & 2 & \\
\hline Yeung and Fung [93] & $\begin{array}{l}\text { Participants sampled for } \\
\text { telephone survey }\end{array}$ & 351 & $\begin{array}{l}\text { No justification or reference } \\
\text { provided }\end{array}$ & Acceptance and denial & 2 & $\begin{array}{l}\text { Only used one item from } \\
\text { each sub-scale, except for } \\
\text { instrumental support, } \\
\text { where one was added; } \\
\text { conducted in Hong Kong, } \\
\text { with no mention of } \\
\text { language used }\end{array}$ \\
\hline
\end{tabular}


Table 4. Summary of factor loading of turning to religion with the other 13 sub-scales from the Brief COPE [1], presented separately for studies that conducted exploratory factor analyses at item level, those that conducted factor analyses at sub-scale level, and those that used factor structures based on previous research or theoretical considerations. Note that Steinhardt and Dolbier [61] reported that turning to religion loaded together with the negatively scored sub-scale substance use, but no entry was made in that case.

\begin{tabular}{|c|c|c|c|c|c|c|c|c|c|c|c|c|c|}
\hline Author & $\begin{array}{l}\text { Active } \\
\text { coping }\end{array}$ & Planning & $\begin{array}{l}\text { Pos. re- } \\
\text { framing }\end{array}$ & $\begin{array}{l}\text { Accept- } \\
\text { ance }\end{array}$ & Humor & $\begin{array}{l}\text { Emo. } \\
\text { support }\end{array}$ & $\begin{array}{l}\text { Instru. } \\
\text { support }\end{array}$ & $\begin{array}{l}\text { Self- } \\
\text { distract. }\end{array}$ & Venting & $\begin{array}{l}\text { Behav. } \\
\text { dis- } \\
\text { engage. }\end{array}$ & Denial & $\begin{array}{l}\text { Sub- } \\
\text { stance } \\
\text { use }\end{array}$ & $\begin{array}{l}\text { Self- } \\
\text { blame }\end{array}$ \\
\hline \multicolumn{14}{|l|}{ Item-level analyses: } \\
\hline Hastings et al. [40] & & & & & & & & & & & 1 & & \\
\hline Lee and Liu [41] & 1 & 1 & 1 & 1 & 0.5 & 1 & & & 0.5 & & & & \\
\hline $\begin{array}{l}\text { Liu and Iwamoto } \\
\text { [42] }\end{array}$ & & & & & & & & & & 1 & 1 & & 1 \\
\hline Paukert et al. [43] & & & & & & & & & & & & 1 & \\
\hline Zelikovsky et al. [16] & & & & & 0.25 & & & & 0.25 & 0.5 & 0.5 & 0.5 & 0.5 \\
\hline Frequency: & $20 \%$ & $20 \%$ & $20 \%$ & $20 \%$ & $15 \%$ & $20 \%$ & $0 \%$ & $0 \%$ & $15 \%$ & $30 \%$ & $50 \%$ & $30 \%$ & $30 \%$ \\
\hline \multicolumn{14}{|c|}{ Sub-scale-level analyses: } \\
\hline $\begin{array}{l}\text { Aitken and Crawford } \\
\text { [52] }\end{array}$ & 1 & 1 & 1 & 1 & & & 1 & & & & & & 1 \\
\hline Bean et al. [53] & 1 & 1 & 1 & 1 & & 1 & 1 & & & & & & \\
\hline $\begin{array}{l}\text { Bellizzi and Blank } \\
\text { [54] }\end{array}$ & 1 & 1 & 1 & 1 & & 1 & 1 & 1 & 1 & & & & \\
\hline Ebert et al. [55] & 1 & 1 & 1 & 1 & & 1 & 1 & & & & & & \\
\hline Farley et al. [18] & & & 1 & 1 & 1 & & & & & & & & \\
\hline Jacobson [56] & 1 & 1 & 1 & & & 1 & 1 & & & & & & \\
\hline Kellezi et al. [57] & & & 1 & & 1 & & & & & & & & 1 \\
\hline Liu et al. [59] & 1 & 1 & 1 & 1 & & 1 & 1 & 1 & & & & & \\
\hline Mugavero et al. [60] & 1 & & 1 & 1 & & 1 & & & & & & & \\
\hline $\begin{array}{l}\text { Schottenbauer et al. } \\
\text { [17] }\end{array}$ & & & & & & 1 & 1 & & & & & & \\
\hline
\end{tabular}


Table 4. Cont.

\begin{tabular}{|c|c|c|c|c|c|c|c|c|c|c|c|c|c|}
\hline \multicolumn{14}{|l|}{$\begin{array}{l}\text { Steinhardt and } \\
\text { Dolbier [61] }\end{array}$} \\
\hline Frequency: & $64 \%$ & $55 \%$ & $91 \%$ & $64 \%$ & $18 \%$ & $64 \%$ & $64 \%$ & $18 \%$ & $9 \%$ & $0 \%$ & $0 \%$ & $0 \%$ & $18 \%$ \\
\hline \multicolumn{14}{|c|}{ Studies using structures based on previous research: } \\
\hline Artinian et al. [94] & 1 & 1 & 1 & 1 & 1 & 1 & 1 & 1 & 1 & & & & \\
\hline Atkinson et al. [77] & 1 & 1 & 1 & 1 & 1 & 1 & 1 & & & & & & \\
\hline $\begin{array}{l}\text { Cartwright et al. } \\
{[106]}\end{array}$ & 1 & 1 & & & & & 1 & & & & & & \\
\hline Cooper et al. [107] & & & 1 & 1 & 1 & 1 & & & & & & & \\
\hline $\begin{array}{l}\text { David and Knight } \\
\text { [91] }\end{array}$ & 1 & 1 & 1 & 1 & 1 & 1 & 1 & & & & & & \\
\hline Gillen [78] & 1 & 1 & 1 & 1 & 1 & 1 & 1 & & & & & & \\
\hline Glass et al. [108] & 1 & 1 & 1 & 1 & 1 & 1 & 1 & & 1 & & & & \\
\hline $\begin{array}{l}\text { Gore-Felton et al. } \\
\text { [97] }\end{array}$ & & & 1 & 1 & & 1 & & & & & 1 & & \\
\hline Gow et al. [92] & & & 1 & 1 & 1 & 1 & & & & & 1 & & \\
\hline Hart et al. [99] & & & & 1 & & 1 & & & & & 1 & & \\
\hline $\begin{array}{l}\text { Hastings and Brown } \\
\text { [79] }\end{array}$ & 1 & 1 & 1 & 1 & 1 & 1 & 1 & & & & & & \\
\hline Hirsch et al. [109] & 1 & 1 & 1 & 1 & 1 & 1 & 1 & & & & & & \\
\hline $\begin{array}{l}\text { Ironson and Kremer } \\
{[100]}\end{array}$ & 1 & 1 & 1 & 1 & & 1 & & & & & & & \\
\hline Kinsinger et al. [101] & 1 & 1 & & & 1 & 1 & & & 1 & & & & \\
\hline Knoll et al. [95] & & & & & & 1 & 1 & & & & & & \\
\hline $\begin{array}{l}\text { Kristiansen et al. } \\
{[80]}\end{array}$ & 1 & 1 & & 1 & 1 & 1 & 1 & & & & & & \\
\hline Krzemien [96] & & & & & & & & 1 & & & 1 & 1 & 1 \\
\hline Lethborg et al. [102] & 1 & 1 & 1 & 1 & 1 & 1 & 1 & & & & & & \\
\hline
\end{tabular}


Table 4. Cont.

\begin{tabular}{|c|c|c|c|c|c|c|c|c|c|c|c|c|c|}
\hline $\begin{array}{l}\text { Lord and Robertson } \\
\text { [81] }\end{array}$ & 1 & 1 & 1 & 1 & 1 & 1 & 1 & & & & & & \\
\hline McIlvane et al. [82] & & & 1 & 1 & 1 & 1 & & & & & & & \\
\hline Meyer [83] & 1 & 1 & 1 & 1 & 1 & 1 & 1 & & & & & & \\
\hline $\begin{array}{l}\text { Moscardino et al. } \\
{[110]}\end{array}$ & & & & & & 1 & 1 & & & & & & \\
\hline O'Brien et al. [84] & 1 & 1 & 1 & 1 & 1 & 1 & 1 & 1 & & & & & \\
\hline O'Connor et al. [85] & 1 & 1 & 1 & 1 & 1 & 1 & 1 & & & & & & \\
\hline $\begin{array}{l}\text { Piazza-Waggoner et } \\
\text { al. [86] }\end{array}$ & 1 & 1 & 1 & 1 & 1 & 1 & 1 & & 1 & & & & \\
\hline Richards et al. [103] & 1 & 1 & 1 & 1 & 1 & 1 & 1 & & & & & & \\
\hline $\begin{array}{l}\text { Rosenberger et al. } \\
\text { [87] }\end{array}$ & 1 & 1 & 1 & 1 & 1 & 1 & & & 1 & & & & \\
\hline Schnider et al. [98] & 1 & 1 & & & & & 1 & & & & & & \\
\hline Schwartz et al. [104] & 1 & 1 & & & & 1 & 1 & & & & & & \\
\hline Steinhardt et al. [88] & 1 & 1 & 1 & 1 & & 1 & 1 & & & & & & \\
\hline $\begin{array}{l}\text { Straughan and } \\
\text { Buckenham [89] }\end{array}$ & 1 & 1 & 1 & 1 & 1 & 1 & 1 & 1 & 1 & & & & \\
\hline Tuncay et al. [111] & 1 & 1 & 1 & 1 & 1 & 1 & 1 & & & & & & \\
\hline $\begin{array}{l}\text { Wichianson et al. } \\
\text { [90] }\end{array}$ & 1 & 1 & 1 & 1 & 1 & 1 & 1 & & & & & & \\
\hline Yeung and Fung [93] & & & & & 1 & 1 & & 1 & 1 & 1 & & 1 & 1 \\
\hline Frequency: & $74 \%$ & $74 \%$ & $71 \%$ & $76 \%$ & $71 \%$ & $91 \%$ & $71 \%$ & $15 \%$ & $21 \%$ & $3 \%$ & $12 \%$ & $6 \%$ & $6 \%$ \\
\hline
\end{tabular}


These results indicate that the methods of factor analysis can have substantial consequences on the conclusions that researchers derive about the role of religious coping in relation to other strategies. The use of the Kaiser-Guttman criterion is known to lead to an inflated number of factors extracted [5,11], although it is impossible to determine to what extent the studies reviewed in the present article have relied in this criterion, since most have indicated the simultaneous use of additional criteria, such as the scree plot or interpretability of the factor solution. Only one of the studies reviewed had used an analysis that is technically appropriate for this type of data: Miyazaki et al. [49] used a robust weighted least squares method with a polychoric correlation matrix. Commonly used techniques, including PCA, are based on the assumption that the data are continuous, which is violated when data are collected using a Likert scale, as is the case with the Brief COPE [1]. When data are ordinal, researchers are instead advised to use an asymptotically distribution free method, such as the weighted least squares [117] or robust weighted least squares method [118].

The use of inappropriate factor analytical techniques in psychological research has been heavily criticized by Bernstein and Teng [37], and may be explainable by researchers' tendency to unquestioning reliance on standard statistical packages without consideration of the assumptions required for using the various estimation methods. Using PCA for non-continuous data tends to inflate the number of extracted factors, even when the scree plot criterion is used in addition to the KaiserGuttman criterion [37]. The practice of combining items to form mini-scales, such as with the subscale-level analyses of the Brief COPE (Table 2), results in variables that are starting to approximate continuous variables more closely. As can be seen in the results of the present review, sub-scale-level analyses extracted a median number of three factors, as opposed to six factors from item-level analyses, which may reflect the increased likelihood of extraction of spurious factors when the latter technique is used. Perhaps some of the loadings of turning to religion with maladaptive coping strategies at item-level analysis may also be the result of this effect: If participants in a sample do not endorse turning to religion or substance use, data from these sub-scales are highly skewed, as for example reported by Kallasmaa and Pulver [119] when using the COPE [10] questionnaire. In that case, when using a PCA with ordinal-level data, it could be possible that these two strategies load together [43] not because of their similarity in content, but simply because of their similarity in response level [37]. Given that religious affiliation is well known to be negatively correlated with use of drugs and alcohol [120], this might be a feasible alternative explanation rather than concluding that these two strategies are genuinely related.

The factor structures used by studies that had not conducted exploratory factor analyses more closely resemble those proposed from sub-scale-level analyses rather than item-level analyses (Table 4). The most striking deviation pertains to the humor sub-scale, which was included into a factor with turning to religion $71 \%$ of the time, whereas the item-level and sub-scale-level analyses reported common loadings only $15 \%$ and $18 \%$ of the time, respectively. With a median number of two factors, studies not conducting exploratory factor analyses tended towards choosing factor structures that were even broader than those that typically emerged from sub-scale-level analyses, where the median number of factors was three. A common way to analyse Brief COPE scores was to combine the scores from the first eight sub-scales into one score (i.e., active coping, planning, positive reframing, acceptance, humor, turning to religion, emotional support, and instrumental support) and the remaining sub-scales into another (Tables 4). Of the 34 studies listed in Table 3, 12 had used this 
approach [77-79,81,83,85,90,91,102,103]. Four of these twelve studies [81,91,102,103] mentioned general theoretical considerations or did not provide any justification or reference. When references were provided, these were frequently to Carver et al. [10], although not always to argue for the use of a four-factor structure, as Carver et al. [10] had proposed for the COPE based on results from their factor analyses. Instead, this reference was sometimes used to justify a two-factor structure, such as adaptive versus problematic coping [94] or adaptive versus maladaptive coping [79], which is most likely based on theoretical comments that Carver et al. [10] had made about the supposed adaptiveness of some of the coping strategies. Atkinson et al. [77] referred to Lunsford et al. [105], although Lunsford et al. themselves had used the individual sub-scales separately. Hastings and Brown [79] and O'Connor et al. [85] referred to Carver et al. [10], and Gillen [78], Hirsch et al. [109] and Wichianson et al. [90] referred to Meyer [83], while Meyer himself had referred to Carver et al. [112]. Studies that did not publish results from exploratory factor analyses of their own dataset (Table 3) therefore did not appear to have been influenced very much by the factor analytical results from studies using the Brief $\mathrm{COPE}$ - or at least the ones that met the criterion of the present review that turning to religion be included.

As can be seen in Table 4, the use of factor structures that used the adaptive versus maladaptive distinction or similar appears to be the dominant approach in studies that did not conduct factor analyses on their own dataset. This distinction, however, has frequently been criticized $[4,121,122]$ with the argument that the effectiveness of the specific coping strategies is context-dependent. With religious coping, variations in effectiveness are also acknowledged by distinguishing between positive or negative religious coping [123,124], where the former involves activities, such as seeking of spiritual support, religious purification and forgiveness, while the latter includes spiritual discontent or punishing God reappraisals. The studies reviewed in the present article do not reveal any clear factor loading pattern for religious coping (Table 4), which may certainly be explainable by variations in the extent of positive versus negative religious coping in different samples.

Tamres et al. [26] noted that religious coping could neither be assigned to problem- nor emotion-focused coping, and the results from the present review echo this statement. For example, religious coping was found to load equally often with problem-focused strategies, such as active coping, than with emotion-focused strategies, such as emotional support (Table 4). One reason suggested to explain such unclear findings is that religious coping might be a unique strategy [27,28], independent of other means of coping. Given the methodological concerns identified above about the use of PCA with ordinal data, reports of religious coping loading as a separate factor do not provide a strong argument for this possibility. Confirmatory factor analyses are a more robust way to investigate the factor structure of religious coping, and of the studies reviewed in the present article, only three used this approach. David and Knight [91] reported acceptable fit indices when religious coping was grouped together with adaptive coping strategies (Table 4), while Knoll et al. [95] and Moscardino et al. [110] reported acceptable fit indices when turning to religion was in the same factor as instrumental and emotional support. A recent study by Krägeloh et al. [125] conducted a series of exploratory and confirmatory factor analyses to compare alternative factor solutions for the Brief COPE [1], and found that, for participants with lower levels of religiosity and spirituality, the best model was when turning to religion was grouped with maladaptive coping strategies. For participants with higher levels of religiosity and spirituality, the best model was when religious coping was grouped with problem- 
focused strategies, followed by emotional coping strategies, and lastly by maladaptive strategies. These findings show that characteristics of the sample can directly affect the way the turning to religion is grouped with secular coping strategies.

The extent to which religious coping may overlap with other strategies or whether it may provide unique coping strategies has been debated in recent research studies. Zwingmann et al. [126] presented data that suggested that the effects of religious coping on psychosocial adjustment in breast cancer patients were fully mediated by non-religious coping strategies, and Pérez et al. [127] found that spiritual striving and depressive symptoms was mediated by acceptance. Burker et al. [128], on the other hand, reported findings that religious coping strategies do not appear to be functionally redundant when predicting stress in lung transplant candidates, as did Schottenbauer et al. [17] in a large survey of respondents self-identifying as Christian. Other evidence for the unique contribution of religious coping strategies comes from Jermann et al. [129], who investigated the effects of mindfulness on depressive symptoms. Mindfulness, which is also a technique central to Buddhism [130], was associated with reduced depressive symptoms, in addition to its indirect effects via other types of coping strategies. Clearly more research is needed to determine if, or which aspect of, religious coping may play a unique and independent function in dealing with stress, and to what extent this may interact with the characteristics of the individual and type of stressful situation.

\subsection{Limitations and conclusions}

The present study outlined the wide range in which turning to religion sub-scale of the Brief COPE [1] is commonly reported to align with other coping strategies. When factor analyses had been conducted using individual items as indicators, religious coping was more likely to load together with maladaptive coping strategies, and more likely with adaptive coping strategies when analyses were conducted at sub-scale level. To a large extent, this range in findings is likely to be due to the diverse and often inappropriate factor analytic techniques used to determine the factor structure of the Brief COPE instrument, therefore unfortunately also affecting the conclusions researchers frequently make about the role of religious coping. PCAs are known to yield unstable factor solutions, especially when used with ordinal-level data [5,11], and conclusions made from such analyses therefore lack validity. While sub-scale-level analyses are more robust, findings were still very variable, with three studies [62-64] reporting that turning to religion formed a separate factor and five studies [21,65-68] reporting lack of sufficiently high factor loadings for religious coping (Table 2). The use of general or uni-dimensional religious coping measures to inform about the role of religion in coping with stress has been criticized [17,131], and in the Brief COPE [1], the scope that the religious coping sub-scale can assess is even further limited by the fact it was reduced to two items, compared to the four items in the COPE. As the development of Brief COPE [1] has not followed recommended guidelines for developing short forms [132], the unstable factor structure of the instrument is not surprising, and it is therefore also uncertain to what extent sub-scale level analyses may be appropriate without prior formal psychometric testing [133]. Because of its brevity, the Brief COPE remains a very popular instrument. However, the present review highlighted how reports from exploratory factor analyses of the Brief COPE have very little value when trying to make general conclusions about the role of religious coping in relation to secular coping methods. Such theoretical questions will need to be 
investigated by using coping instruments that more specifically and comprehensively assess the nature of religious coping, such as the RCOPE [123].

Several limitations of the present study need to be acknowledged, as they leave unaddressed some potential alternative explanations. Firstly, the only criterion used for inclusion of articles in the present review was completeness of information about the use of the Brief COPE [1] and particularly the turning to religion sub-scale. As outlined in the methods section above, 78 studies could therefore not be analyzed. No a priori criterion of methodological rigour was applied, and therefore the range in findings could also be a reflection of the range in methodological rigor of the reviewed studies. Secondly, the range in the target populations varied enormously across the reviewed studies. As Krägeloh et al. [125] showed, this diversity may also be a contributing factor to the wide range of reports about the role of religious coping in relation to other coping mechanisms. Finally, to contain the already large scope of the present review, no analyses were shown how the outcomes from exploratory factor analyses differed for studies that used the situational and those that used the dispositional version of the questionnaire. In the dispositional version, Likert-scale descriptors are worded in terms of participants' usual tendency to use a particular coping strategy (e.g. "I usually don't do this at all") instead of referring to specific events that they are being asked to recall (e.g. "I've been doing this a lot"). Of the 36 studies shown in Tables 1 and 2, eight studies used the dispositional version $[16,18,50,52,55,61,62,67]$, and the results were equally varied as those of the remaining studies that used the situational version. To a large extent, any differences would be confounded by differences in sample characteristics, since the situational version tends to be administered to participants who have or are currently encountering specific stressors, while the dispositional version tends to be administered to target populations with no specific common stressor, such as convenience samples [62] or university students [50,55,61]. Further research is therefore necessary to explore whether responses to religious coping questionnaires framed in a dispositional format may result in different factor solutions than those in situational formats.

\section{References}

1. Carver, C.S. You want to measure coping but your protocol's too long: Consider the Brief COPE. Int. J. Beh. Med. 1997, 4, 92-100.

2. Skinner, E.A.; Zimmer-Gembeck, M.J. The development of coping. Annu. Rev. Psychol. 2007, $58,119-144$.

3. Folkman, S.; Moskowitz, J.T. Coping: Pitfalls and promise. Annu. Rev. Psychol. 2004, 55, 745-774.

4. Lazarus, R.S.; Folkman, S. Stress Appraisal and Coping; Springer: New York, NY, USA, 1984.

5. Parker, J.D.A.; Endler, N.S. Coping with coping assessment: A critical review. Euro. J. Perso. 1992, 6, 321-344.

6. Skinner, E.A.; Edge, K.; Altman, J.; Sherwood, H. Searching for the structure of coping: A review and critique of category systems for classifying ways of coping. Psychol. Bull. 2003, 129, 216-269.

7. Billings, A.G.; Moos, R.H. The role of coping responses and social resources in attenuating the stress of life events. J. Behav. Med. 1981, 4, 139-157. 
8. Endler, N.S.; Parker, J.D.A. Multidimensional assessment of coping: A critical evaluation. J. Person. Soc. Psychol. 1990, 58, 844-854.

9. Coolidge, F.L.; Segal, D.L.; Hook, J.N.; Stewart, S. Personality disorders and coping among anxious older adult. J. Anxiety Disord. 2000, 14, 157-172.

10. Carver, C.S.; Scheier, M.F.; Weintraub, J.K. Assessing coping strategies: A theoretically based approach. J. Person. Soc. Psychol. 1989, 56, 267-283.

11. Cliff, N. The eigenvalue-greater-than-one rule and the reliability of components. Psychol. Bull. 1988, 103, 276-279.

12. Ingledew, D.K.; Hardy, L.; Cooper, C.L.; Jemal, H. Health behaviours reported as coping strategies: A factor analytical study. Brit. J. Health Psychol. 1996, 1, 263-281.

13. Lyne, K.; Roger, D. A psychometric re-assessment of the COPE questionnaire. Person. Individ. Diff. 2000, 29, 321-335.

14. Zuckerman, M.; Gagne, M. The COPE revised: Proposing a 5-factor model of coping strategies. J. Res. Person. 2003, 37, 169-204.

15. Radat, F.; Lantéri-Minet, M.; Nachit-Ouinekh, F.; Massiou, H.; Lucas, C.; Pradalier, A.; Mercier, F.; El Hasnaoui, A. The GRIM2005 study of migraine consultation in France. III: Psychological features of subjects with migraine. Cephalalgia. 2009, 29, 338-350.

16. Zelikovsky, N.; Schast, A.P.; Jean-Francois, D. Parent stress and coping: Waiting for a child to receive a kidney transplant. J. Clin. Psychol. Med. Settings. 2007, 14, 320-329.

17. Schottenbauer, M.A.; Klimes-Dougan, B.; Rodriguez, B.F.; Arnkoff, D.B.; Glass, C.R.; Lasalle, V.H. Attachment and affective resolution following a stressful event: General and religious coping as possible mediators. Ment. Health Relig. Cult. 2006, 9, 448-471.

18. Farley, T.; Galves, A.; Dickinson, L.M.; de Jesus Diaz Perez, M. J. Immigrant Health. 2005, 7, 213-220.

19. Brain, K.; Henderson, B.J.; Tyndel, S.; Bankhead, C.; Watson, E.; Clements, A.; Austoker, J. On behalf of the PIMMS Study Management Group. Predictors of breast cancer-related distress following mammography screening in younger women on a family history breast screening programme. Psycho-Oncology 2008, 17, 1180-1188.

20. Perczek, R.; Carver, C.S.; Price, A.A.; Pozo-Kaderman, C. Coping, mood, and aspects of personality in Spanish translation and evidence of convergence with English versions. J. Person. Assess. 2000, 74, 63-87.

21. Lawrence, J.W.; Fauerbach, J.A. Personality, coping, chronic stress, social support and PTSD symptoms among adult burn survivors - a path analysis. J. Burn Care Rehabil. 2003, 24, 63-72.

22. Welbourne, J.L., Eggerth, D.; Hartley, T.A.; Andrew, M.E.; Sanchez, F. Coping strategies in the workplace: Relationships with attributional style and job satisfaction. J. Vocat. Behav. 2007, 70, 312-325.

23. Koenig, H.G.; McCullough, M.E.; Larson, D.B. Handbook of Religion and Health; Oxford University Press: Oxford, UK, 2001.

24. Larson, D.B.; Larson, S.S. Spirituality's potential relevance to physical and emotional health: A brief review of quantitative research. J. Psychol. Theol. 2003, 31, 37-51.

25. Seybold, K.S.; Hill, P.C. The role of religion and spirituality in mental and physical health. Curr. Direct. Psychol. Sci. 2001, 10, 21-24. 
26. Tamres, L.K.; Janicki, D.; Helgeson, V.S. Sex differences in coping behavior: A meta-analytic review and an examination of relative coping. Person. Soc. Psychol. Rev. 2002, 6, 2-30.

27. Cook, S.W.; Heppner, P.P. A psychometric study of three coping measures. Educ. Psychol. Meas. 1997, 57, 906-923.

28. Hudek-Knežević, J.; Kardum, I.; Vukmirović, Ž. The structure of coping styles. A comparative study of Croatian sample. Euro. J. Person. 1999, 13, 149-161.

29. Pack, R.; Krishnamurthy, G.; Cottrell, L.; Stanton, B.; D’Alessandri, D.; Burns, J. Caregiver predictors of adolescent inhalant abuse in rural Appalachia. Am. J. Health Behav. 2005, 29, 331-341.

30. Pritchard, M.E.; Wilson, G.S. Using emotional and social factors to predict student success. J. Coll. Stud. Develop. 2003, 44, 18-28.

31. Strug, D.L.; Mason, S.E.; Auerbach, C. How older Hispanic immigrants in New York City cope with current traumatic stressors: Practice implications. J. Geront. Soc. Work. 2009, 52, 503-516.

32. Wilson, G.S.; Pritchard, M.E.; Schaffer, J. Athletic status and drinking behavior in college students: The influence of gender and coping styles. J. Am. Coll. Health. 2004, 52, 269-273.

33. Bishop, A.J. Age and gender differences in adaptation and subjective well-being of older adults residing in monastic religious communities. Pastoral Psychol. 2006, 55, 131-143.

34. Fogel, J.; Albert, S.M.; Schnabel, F.; Ditkoff, B.A.; Neugut, A.I. Internet use and social support in women with breast cancer. Health Psychol. 2002, 21, 398-404.

35. Fogel, J.; Albert, S.M.; Schnabel, F.; Ditkoff, B.A.; Neugut, A.I. Racial/ethnic differences and potential psychological benefits in use of the internet by women with breast cancer. PsychoOncology 2003, 12, 107-117.

36. Brown, T.A. Confirmatory Factor Analysis for Applied Research; Guildford Press: New York, NY, USA, 2006.

37. Bernstein, I.H.; Teng, G. Factoring items and factoring scales are different: Spurious evidence for multidimensionality due to item categorization. Psychol. Bull. 1989, 105, 467-477.

38. Henderson, B. J.; Tyndel, S.; Brain, K.; Clements, A.; Bankhead, C.; Austoker, J.; Watson, E., on behalf of the PIMMS Study Management Group. Factors associated with breast cancer-specific distress in younger women participating in a family history mammography screening programme. Psycho-Oncology 2008, 17, 74-82.

39. Lucas, C.; Lantéri-Minet, M.; Massiou, H.; Nachit-Ouinekh, F.; Pradalier, A.; Mercier, F.; El Hasnaoui, A.; Radat, F. The GRIM2005 study of migraine consultation in France II. Psychological factors associated with treatment response to acute headache therapy and satisfaction in migraine. Cephalalgia 2007, 27, 1398-1407.

40. Hastings, R.P.; Kovshoff, H.; Brown, T.; Ward, N.J.; degli Espinosa, F.; Remington, B. Coping strategies in mothers and fathers of preschool and school-age children with autism. Autism 2005, 9, 377-391.

41. Lee, R.M.; Liu, H.-T.T. Coping with intergenerational family conflict: Comparison of Asian American, Hispanic, and European American college students. J. Counsel. Psychol. 2001, 48, 410-419. 
42. Liu, W.M.; Iwamoto, D.K. Conformity to masculine norms, Asian values, coping strategies, peer group influences and substance use among Asian American men. Psychol. Men Masculinity 2001, 8, 25-39.

43. Paukert, A.L.; LeMaire, A.; Cully, J.A. Predictors of depressive symptoms in older veterans with heart failure. Age Ment. Health. 2009, 13, 601-610.

44. Gorsuch, R.L. Factor Analysis; Erlbaum: Hillsdale, NJ, USA, 1983.

45. Cicognani, E.; Pietrantoni, L.; Palestini, L.; Prati, G. Emergency workers' quality of life: The protective role of sense of community, efficacy beliefs and coping strategies. Soc. Indic. Res. 2009, 94, 449-463.

46. Fillion, L.; Kovacs, A.H.; Gagnon, P.; Endler, N.S. Validation of the shortened COPE for use with breast cancer patients undergoing radiation therapy. Curr. Psychol. 2002, 21, 17-34.

47. Fletcher, K.E.; Clemow, L.; Peterson, B.A.; Lemon, S.C.; Estabrook, B.; Zapka, J.G. A path analysis of factors associated with distress among first-degree female relatives of women with breast cancer diagnosis. Health Psychol. 2006, 25, 413-424.

48. Heydecke, G.; Tedesco, L.A.; Kowalski, C.; Inglehart, M.R. Complete dentures and oral healthrelated quality of life - do coping styles matter? Comm. Dent. Oral Epidemiol. 2004, 32, 297-306.

49. Miyazaki, Y.; Bodenhorn, N.; Zalaquett, C.; Ng, K.-M. Factorial structure of Brief COPE for international students attending U.S. colleges. Coll. Stud. J. 2008, 42, 795-806.

50. Ribeiro, J.L.P.; Rodrigues, A.P. Questões acerca do coping: A propósito do estudo de adaptação do Brief COPE. Psicologia, Saúde Doenças 2004, 5, 3-15.

51. Udipi, S.; Veach, P.M.; Kao, J.; LeRoy, B.S. The psychic costs of emphatic engagement: Personal and demographic predictors of genetic counselor compassion fatigue. J. Genet. Counsel. 2008, 17, 459-471.

52. Aitken, A.; Crawford, L. Coping with stress: Dispositional coping strategies of project managers. Int. J. Project Manage. 2007, 25, 666-673.

53. Bean, M.K.; Gibson, D.; Flattery, M.; Duncan, A.; Hess, M. Psychosocial factors, quality of life, and psychological distress: Ethnic differences in patients with heart failure. Progr. Card. Nurs. 2009, 24, 131-140.

54. Bellizzi, K.M.; Blank, T.O. Predicting posttraumatic growth in breast cancer survivors. Health Psychol. 2006, 25, 47-56.

55. Ebert, S.A.; Tucker, D.C.; Roth, D.L. Psychological resistance factors as predictors of general health status and physical symptom reporting. Psychol. Health Med. 2002, 7, 363-375.

56. Jacobson, J.M. Compassion fatigue, compassion satisfaction, and burnout: Reactions among employee assistance professionals providing workplace crisis intervention and disaster management services. J. Workplace Behav. Health. 2006, 21, 133-152.

57. Kellezi, B.; Reicher, S.; Cassidy, C. Surviving the Kosovo conflict: A study of social identity, appraisal of extreme events, and mental well-being. Appl. Psychol. Int. Rev. 2009, 58, 59-83.

58. Kershaw, T.; Northouse, L.; Kritpracha, C.; Schafenacker, A.; Mood, D. Coping strategies and quality of life in women with advanced breast cancer and their family caregivers. Psychol. Health.

2004, 19, 139-155. 
59. Liu, H.; Feurer, I.D.; Dwyer, K.; Shaffer, D.; Pinson, C.W. Effects of clinical factors on psychosocial variables in renal transplant recipients. J. Adv. Nurs. 2009, 65, 2585-2596.

60. Mugavero, M.J.; Raper, J.L.; Reif, S.; Whetten, K.; Leserman, J.; Thielman, N.M.; Pence, B.W. Overload: Impact of incident stressful events on antiretroviral medication adherence and virologic failure in a longitudinal, multisite human immunodeficiency virus cohort study. Psychosom. Med. 2009, 71, 920-926.

61. Steinhardt, M.; Dolbier, C. Evaluation of a resilience intervention to enhance coping strategies and protective factors and decrease symptomatology. J. Am. Coll. Health. 2008, 56, 445-453.

62. Saroglou, V.; Anciaux, L. Liking sick humor: Coping styles and religion as predictors. Humor. 2004, 17, 257-277.

63. Weininger, C.F.; Shalev, A.Y.; Ofek, H.; Freedman, S.; Weissman, C.; Einav, S. Posttraumatic stress disorder among hospital surgical physicians exposed to victims of terror: A prospective, controlled questionnaire survey. J. Clin. Psychiatry 2006, 67, 890-896.

64. Wood, R.L.L.; Rutterford, N.A. Demographic and cognitive predictors of long-term psychosocial outcome following traumatic brain injury. J. Int. Neuropsychol. Soc. 2006, 12, 350-358.

65. Kershaw, T.S.; Mood, D.W.; Newth, G.; Ronis, D.L.; Sanda, M.G.; Vaishampayan, U.; Northouse, L. Longitudinal analysis of a model to predict quality of life in prostate cancer patients and their spouses. Ann. Behav. Med. 2008, 36, 117-128.

66. Myaskovsky, L.; Dew, M.A.; Switzer, G.E.; McNulty, M.L.; DiMartini, A.F.; McCurry, K.R. Quality of life and coping strategies among lung transplant candidates and their family caregivers. Soc. Sci. Med. 2005, 60, 2321-2332.

67. Ng, S.K.S.; Leung, W.K. A community study on the relationship between stress, coping, affective dispositions and periodontal attachment loss. Comm. Dent. Oral Epidemiol. 2006, 34, 252-266.

68. Yang, H.-C.; Brothers, B.M.; Andersen, B.L. Stress and quality of life in breast cancer recurrence: Moderation or mediation of coping? Ann. Behav. Med. 2008, 35, 188-197.

69. Cooper, C.; Katona, C.; Orrell, M.; Livingston, G. Coping strategies and anxiety in caregivers of people with Alzheimer's disease: The LASER-AD study. J. Affect. Disord. 2006, 90, 15-20.

70. Cooper, C.; Katona, C.; Orrell, M.; Livingston, G. Coping strategies, anxiety and depression in caregivers of people with Alzheimer's disease. Int. J. Ger. Psychiatry 2008, 23, 929-936.

71. Cooper, C.; Owens, C.; Katona, C.; Livingston, G. Attachment style and anxiety in carers of people with Alzheimer's disease: Results from the LASER-AD study. Int. Psychoger. 2008, 20, 494-507.

72. Gillen, G. A comparison of situational and dispositional coping after a stroke. Occup. Therap. Ment. Health. 2006, 22, 31-59.

73. Gore-Felton, C.; Vosvick, M.; Bendel, T.; Koopman, C.; Das, B.; Israelski, D.; Herrera, M.; Litzenberg, K.; Spiegel, D. Correlates of sexually transmitted disease infection among adults living with HIV. Int. J. STD AIDS 2003, 14, 539-546.

74. Gow, K.; Warren, C.; Anthony, D.; Hinschen, C. Retention and intentions to quit among Australian male apprentices. Educ. Train. 2008, 50, 216-230.

75. Pence, B.W.; Thielman, N.M.; Whetten, K.; Ostermann, J.; Kumar, V.; Mugavero, M.J. Coping strategies and patterns of alcohol and drug use among HIV-infected patients in the United States southeast. AIDS Patient Care STDs 2008, 11, 869-877. 
76. Piazza-Waggoner, C.; Adams, C.D.; Muchant, D.; Wilson, N.W.; Hogan, M. B. Coping and adjustment in children with primary immunodeficiency disorders and kidney diseases: The role of illness severity. Child. Health Care 2008, 37, 210-224.

77. Atkinson, J.H.; Higgins, J.A.; Vigil, O.; Dubrow, R.; Remien, R.H.; Steward, W.T.; Casey, C.Y.; Sikkema, K.J.; Correale, J.; Ake, C.; et al. Psychiatric context of acute/early HIV infection. The NIMH multisite acute HIV infection study: IV. AIDS Behavior 2009, 13, 1061-1067.

78. Gillen, G. Coping during inpatient stroke rehabilitation: An exploratory study. Am. J. Occup. Ther. 2006, 60, 136-145.

79. Hastings, R.P.; Brown, T. Coping strategies and the impact of challenging behaviors on special educators' burnout. Ment. Retard. 2002, 40, 148-156.

80. Kristiansen, E.; Roberts, G.C.; Abrahamsen, F.E. Achievement involvement and stress coping in elite wrestling. Scand. J. Med. Sci. Sports 2008, 18, 526-538.

81. Lord, S.; Robertson, N. The role of patient appraisal and coping in predicting distress in IVF. J. Reprod. Infant Psychol. 2005, 23, 319-332.

82. McIlvane, J.M.; Popa, M.A.; Robinson, B.; Houseweart, K.; Haley, W.E. Perceptions of illness, coping, and well-being in persons with mild cognitive impairment and their care partners. Alzheimer Dis. Assoc. Disord. 2008, 22, 284-292.

83. Meyer, B. Coping with severe mental illness: Relations of the Brief COPE with symptoms, functioning, and well-being. J. Psychopathol. Behav Assess. 2001, 23, 265-277.

84. O’Brien, M.P.; Zinberg, J.L.; Bearden, C.E.; Daley, M.; Niendam, T.A.; Kopelowicz, A.; Cannon, T.D. Psychoeducational multi-family group treatment with adolescents at high risk for developing psychosis. Early Interv. Psychiatry 2007, 1, 325-332.

85. O'Connor, S.M.; Jardine, A.G.; Millar, K. The prediction of self-care behaviors in end-stage renal disease patients using Leventhal's self-regulatory model. J. Psychosom. Res. 2008, 65, 191-200.

86. Piazza-Waggoner, C.; Adams, C.D.; Muchant, D.; Wilson, N.; Hogan, M.B. Children with primary immunodeficiency disorders: Family functioning, coping strategies, and behavioural functioning. Child. Health Care 2006, 35, 191-208.

87. Rosenberger, P.H.; Ickovics, J.R.; Epel, E.S.; D’Entremont, D.; Jokl, P. Physical recovery in arthroscopic knee surgery: Unique contributions of coping behaviors to clinical outcomes and stress reactivity. Psychol. Health 2004, 19, 307-320.

88. Steinhardt, M.A.; Mamerow, M.M.; Brown, S.A.; Jolly, C.A. A resilience intervention in African American adults with Type 2 diabestes - a pilot study of efficacy. Diabetes Educ. 2009, 35, 274-284.

89. Straughan, H.; Buckenham, M. In-sight: An evaluation of user-led, recovery-based, holistic group training for bipolar disorder. J. Public Ment. Health 2006, 5, 29-43.

90. Wichianson, J.R.; Bughi, S.A.; Unger, J.B.; Spruijt-Metz, D.; Nguyen-Rodriguez, S.T. Perceived stress, coping and night-eating in college students. Stress Health 2009, 25, 235-240.

91. David, S.; Knight, B.G. Stress and coping among gay men: Age and ethnic differences. Psychol. Aging. 2008, 23, 62-69.

92. Gow, K.; Hinschen, C.; Anthony, D.; Warren, C. Work expectations and other factors influencing male apprentices' intentions to quit their trade. Asia Pac. J. Hum. Resour. 2008, 46, 99-121. 
93. Yeung, D.Y.-L.; Fung, H.H. Age differences in coping and emotional responses toward SARS: A longitudinal study of Hong Kong Chinese. Aging Ment. Health. 2007, 11, 579-587.

94. Artinian, N.T.; Abrams, J.; Keteyian, S.J.; Franks, M.M.; Franklin, B.; Pienta, A.; Tkatch, R.; Cuff, L.; Alexander, P.; Schwartz, S. Correlates of depression at baseline among African Americans enrolled in cardiac rehabilition. J. Cardiopul. Rehabil. Prev. 2009, 29, 24-31.

95. Knoll, N.; Rieckmann, N.; Schwarzer, R. Coping as a mediator between personality and stress outcomes: A longitudinal study with cataract surgery patients. Euro. J. Person. 2005, 19, 229-247.

96. Krzemien, D. Estilos de personalidad y afrontamiento situacional frente al envejecimiento en la mujer. R. Interam. Psicol. 2007, 41, 139-150.

97. Gore-Felton, C.; Koopman, C.; Turner-Cobb, J.M.; Durán, R.; Israelski, D.; Spiegel, D. The influence of social support, coping and mood on sexual risk behavior among HIV-positive men and women. J. Health Psychol. 2002, 7, 713-722.

98. Schnider, K.R.; Elhai, J.D.; Gray, M.J. Coping style use predicts posttraumatic stress and complicated grief symptom severity among college students reporting a traumatic loss. J. Counsel. Psychol. 2007, 54, 344-350.

99. Hart, S.; Gore-Felton, C.; Maldonado, J.; Lagana, L.; Blake-Mortimer, J.; Israelski, D.; Koopman, C.; Spiegel, D. The relationship between pain and coping styles among HIV-positive men and women. Psychol. Health. 2000, 15, 869-879.

100. Ironson, G.; Kremer, H. Spiritual transformation, psychological well-being, health, and survival in people with HIV. Int. J. Psychiatry Med. 2009, 39, 263-281.

101. Kinsinger, D.P.; Penedo, F.J.; Antoni, M.H.; Dahn, J.R.; Lechner, S.; Schneiderman, N. Psychosocial and sociodemographic correlates of benefit-finding in men treated for localized prostate cancer. Psych-Oncology 2006, 15, 954-961.

102. Lethborg, C.; Aranda, S.; Cox, S.; Kissane, D. To what extent does meaning mediate adaptation to cancer? The relationship between physical suffering, meaning in life, and connection to others in adjustment to cancer. Pall. Support. Care 2007, 5, 377-388.

103. Richards, H.L.; Ling, T.C.; Evangelou, G.; Brooke, R.C.C.; Fortune, D.G.; Rhodes, L.E. Evidence of high levels of anxiety and depression in polymorphic light eruption and their association with clinical and demographic variables. Brit. J. Dermatol. 2008, 159, 439-444.

104. Schwartz, R.M.; Hogben, M.; Liddon, N.; Augenbraun, M.; McCormack, W.M.; Rubin, S.; Wilson, T.E. Coping with a diagnosis of $\mathrm{C}$ trachomatis or $\mathrm{N}$ gonorrhoeae: Psychosocial and behavioural correlates. J. Health Psychol. 2008, 13, 921-929.

105. Lunsford, S.L.; Simpson, K.S.; Chavin, K.D.; Hildebrand, L.G.; Miles, L.G.; Shilling, L.M.; Smalls, G.R.; Baliga, P.K. Racial differences in coping with the need for kidney transplantation and willingness to ask for live organ donation. Am. J. Kidney Dis. 2006, 47, 324-331.

106. Cartwright, T.; Endean, N.; Porter, A. Illness perceptions, coping and quality of life in patients with alopecia. Brit. J. Dermatol. 2009, 160, 1034-1039.

107. Cooper, C.; Katona, C.; Livingston, G. Validity and reliability of the Brief COPE in carers of people with dementia. J. Nerv. Ment. Dis. 2008, 196, 838-843. 
108. Glass, K.; Flory, K.; Hankin, B.L.; Kloos, B.; Turecki, G. Are coping strategies, social support, and hope associated with psychological distress among Hurricane Katrina survivors? J. Soc. Clin. Psychol. 2009, 28, 779-795.

109. Hirsch, J.D.; Do, A.H.; Hollenbach, K.A.; Manoguerra, A.S.; Adler, D.S. Students' health-related quality of life across the preclinical pharmacy curriculum. Am. J. Pharm. Educ. 2009, 73, 1-6.

110. Moscardino, U.; Scrimin, S.; Capello, F.; Altoè, G.; Axia, G. Psychological adjustment of adolescents 18 months after the terrorist attack in Beslan, Russia: A cross-sectional study. J. Clin. Psychiatry 2008, 69, 854-859.

111. Tuncay, T.; Musabak, I.; Gok, D.E.; Kutlu, M. The relationship between anxiety, coping strategies and characteristics of patients with diabetes. Health Qual. Life Outcomes 2008, 6, 79-87.

112. Carver, C.S.; Pozo, C.; Harris, S.D.; Noriega, V.; Scheier, M.F.; Robinson, D.S.; Ketcham, A.S.; Moffat, F.L.; Clark, K.C. How coping mediates the effect of optimism on distress: A study of women with early stage breast cancer. J. Person. Soc. Psychol. 1993, 65, 375-390.

113. Feaster, D.J.; Szapocznik, J. Interdependence of stress processes among African American family members: influence of HIV serostatus and a new infant. Psychol. Health 2002, 17, 339-363.

114. Spirito, A. Commentary: Pitfalls in the use of brief screening measures of coping. J. Ped. Psychol. 1996, 21, 573-575.

115. Stephens, M.A.P.; Zautra, A.J.; Druley, J.A. Older adults' recovery from surgery for osteoarthritis of the knee: Psychosocial resources and contraints as predictors of outcomes. Health Psychol. 2002, 21, 377-383.

116. Tuna, M.E. Cross-Cultural Differences in Coping Strategies as the Predictors of University Adjustment of Turkish and U.S. Students. PhD Thesis, Middle East Technical University, Ankara, Turkey, 2 December 2003.

117. Jöreskog, K.G. New developments in LISREL: Analysis of ordinal variables using polychoric correlations and weighted least squares. Qual. Quant. 1990, 24, 387-404.

118. Flora, D.B.; Curran, P.J. An empirical evaluation of alternative methods of estimation for confirmatory factor analysis with ordinal data. Psychol. Meth. 2004, 9, 466-491.

119. Kallasmaa, T.; Pulver, A. The structure and properties of the Estonian COPE inventory. Person. Individ. Diff. 2000, 29, 881-894.

120. Gorsuch, R.L. Religious aspects of substance abuse and recovery. J. Soc. Iss. 1995, 51, 65-83.

121. David, D.; Montgomery, G.H.; Bovbjerg, D.H. Relations between coping responses and optimism-pessimism in predicting anticipatory psychological distress in surgical breast cancer patients. Person. Individ. Diff. 2006, 40, 203-213.

122. Lazarus, R.S. Toward better research on stress and coping. Am. Psychol. 2000, 55, 665-673.

123. Pargament, K.I.; Koenig, H.G.; Perez, L.M. The many methods of religious coping: Development and initial validation of the RCOPE. J. Clin. Psychol. 2000, 56, 519-543.

124. Pargament, K.I.; Smith, B.W.; Koenig, H.G.; Perez, L. Patterns of positive and negative religious coping with major life stressors. J. Sci. Study Relig. 1998, 37, 710-724.

125. Krägeloh, C.U.; Chai, P.P.M.; Shepherd, D.; Billington, R. How religious coping is used relative to other coping strategies depends on the individual's level of religiosity and spirituality. J. Rel. Health. 2011, doi: 10.1007/s10943-010-9416-x. 
126.Zwingmann, C.; Wirtz, M.; Müller, C.; Körber, J.; Murken, S. Positive and negative religious coping in German breast cancer patients. J. Behav. Med. 2006, 29, 533-547.

127. Pérez, J.E.; Chartier, M.; Koopman, C.; Vosvick, M.; Gore-Felton, C.; Spiegel, D. Spiritual striving, acceptance coping, and depressive symptoms among adults living with HIV/AIDS. J. Health Psychol. 2009, 14, 88-97.

128. Burker, E.J.; Evon, D.M.; Sedway, J.A.; Egan, T. Religious and non-religious coping in lung transplant candidates: Does adding God to the picture tell us more? J. Behav. Med. 2005, 28, 513-526.

129. Jermann, F.; Billieux, J.; Larøi, F.; d'Argembeau, A.; Bondolfi, G.; Zermatten, A.; Van der Linden, M. Mindful attention awareness scale (MAAS): Psychometric properties of the French translation and exploration of its relations with emotion regulation strategies. Psychol. Assess. 2009, 21, 506-514.

130. Phillips, R.E.; Cheng, C.M.; Pargament, K.I.; Oemig, C.; Colvin, S.D.; Abarr, A.N.; Dunn, M.W.; Reed, A.S. Spiritual coping in American Buddhists: An exploratory study. Int. J. Psychol. Relig. 2009, 19, 231-243.

131. Lavery, M.E.; O'Hea, E.L. Religious/spirtual coping and adjustment in individuals with cancer: Unanswered questions, important trends, and future directions. Ment. Health Relig. Cult. 2010, $13,55-65$.

132. Smith, G.T.; McCarthy, D.M.; Anderson, K.G. On the sins of short-form development. Psychol. Assess. 2000, 12, 102-111.

133. Little, T.D.; Cunningham, W.A.; Shahar, G. To parcel or not to parcel: Exploring the question, weighing the merits. Struct. Equation Model. 2002, 9, 151-173.

(C) 2011 by the authors; licensee MDPI, Basel, Switzerland. This article is an open access article distributed under the terms and conditions of the Creative Commons Attribution license (http://creativecommons.org/licenses/by/3.0/). 